\title{
MICROENCAPSULACIÓN DE ACEITE DE POMELO POR GELIFICACIÓN IONICA Y EXTRUSIÓN
}

\section{Microencapsulation of grapefruit oils by ionic gelation and extrusion}

Presentación: 17/10/2019

\section{Doctorando:}

\section{Liliana Mariel Cáceres}

Centro de Investigación en Química Orgánica Biológica (QUIMOBI), UTN-FRRe, French 414, H3500CHJ, Resistencia, Chaco, Argentina. Instituto de Modelado e Innovación Tecnológica (IMIT-CONICET), Corrientes, Argentinacorreoelectronico@correo.com Imarielc@yahoo.com.ar; macaceres@frre.utn.edu.ar

\section{Director/es:}

\section{Ester Chamorro}

\section{Resumen}

La microencapsulación es definida como una tecnología de empaquetamiento, aplicada con éxito en la industria alimentaria, biotecnológica y farmacéutica. Se la utiliza como método de protección de principios activos sensibles a factores externos, como los aceites esenciales. De esta manera, estos compuestos pueden ser incorporados a distintos productos donde se lleva a cabo su liberación controlada. Se aprovecha así sus propiedades antimicrobianas, conservantes, saborizantes o aromatizantes, entre otras. En este trabajo se optimizó el proceso de microencapsulación del aceite esencial de pomelo con alginato de sodio por gelificación iónica externa acoplada a una extrusión. Se utilizó alginato de sodio al $1 \% \mathrm{p} / \mathrm{v}$ y carga de aceite esencial del 2\% p/p. Se determinó el punto óptimo con concentración de cloruro de calcio del $10 \% \mathrm{p} / \mathrm{v}$ con tiempo de reticulación de 45 minutos. En estas condiciones la eficiencia media fue de $95,89 \pm 0,04 \%$, y el rendimiento medio de $56,87 \pm 1,2 \%$.

Palabras claves: Microencapsulación, Alginato, Aceite esencial, Aceite de pomelo

\begin{abstract}
Microencapsulation is defined as a packaging technology, successfully applied in the food, biotechnology and pharmaceutical industry. It is used as a method of protection of active ingredients sensitive to external factors, such as essential oils. In this way, these compounds can be incorporated into different products where controlled release takes place. It takes advantage of its antimicrobial, preservative, flavoring or flavoring properties, among others. In this work the microencapsulation process of grapefruit essential oil with sodium alginate was optimized by external ionic gelation coupled to an extrusion. $1 \% \mathrm{w} / \mathrm{v}$ sodium alginate and $2 \% \mathrm{w} / \mathrm{w}$ essential oil load were used. The optimum point was determined with calcium chloride concentration of $10 \% \mathrm{w} / \mathrm{v}$ with cross-linking time of 45 minutes. Under these conditions the average efficiency was $95.89 \pm 0.04 \%$, and the average yield $56.87 \pm 1.2 \%$.
\end{abstract}

Keywords: Microencapsulation, Alginate, Essential oils, Grapefruit oils.

\section{Introducción}

Los aceites esenciales son muy utilizados como aditivos en la industria alimentaria (Benavidez et al., 2016). Sin embargo, presentan alta volatilidad e inestabilidad química en presencia de aire, luz, humedad y altas temperaturas. Una solución a este problema es microencapsular estos ingredientes antes de su uso en alimentos o bebidas para impedir su degradación o pérdida de aroma durante el procesamiento y almacenamiento (Jun-Xia et al., 2012). El proceso de microencapsulación consiste en generar una matriz polimérica que recubre el aceite esencial, al cual 
protege, y luego permite su liberación controlada en determinadas condiciones (Hosseini et al., 2013). Los polímeros utilizados generalmente son hidrocoloides (Lupo Pasin et al., 2012), como el alginato de sodio, con propiedades coloidales únicas y una composición química que permiten la gelificación iónica (Smidsrod y Draget, 1996). Este método es versátil y simple y se adosa una extrusión (Chan, 2011) por medio de una bomba jeringa (Pasukamonset et al., 2016) para microencapsular aceites esenciales.

El objetivo de este trabajo es microencapsular el aceite de pomelo por gelificación iónica y extrusión y optimizar el proceso.

\section{Metodología:}

\subsection{Reactivos}

Se usó alginato de sodio (Aldrich, 39\% de ácido gulurónico y 61\% ácido manurónico, PM de 120000 a 190000 $\mathrm{g} / \mathrm{mol}$ ), cloruro de calcio anhidro (Cicarelli p.a.). El aceite de pomelo, provisto por Mager (Corrientes, Argentina), contiene 93\% g/g de limoneno y en estudios anteriores se demuestra que inhibe el crecimiento de bacterias Escherichia coli, Staphylococcus aureus, Lactococcus lactis subsp. lactis, Lactococcus lactis subsp. diacetylactis, Leuconostoc mesenteroides subsp. dextranicum y Lactobacillus plantarum (Vasek et al., 2015).

\subsection{Obtención de las microcápsulas}

Se disolvió alginato de sodio en agua destilada $\left(0.2 \mu \mathrm{S}\right.$ de conductividad a $\left.40^{\circ} \mathrm{C}\right)$ para formar una solución al $1 \%$ $\mathrm{p} / \mathrm{p}$. Se midió luego la viscosidad de la solución con de alginato, con un viscosímetro digital marca Ni-Run, modelo NDJ-8S, utilizando el husillo $\mathrm{N}^{\circ} 0$. Se dejó una hora en reposo para eliminar las burbujas de aire. Se prepararon las soluciones de cloruro de calcio anhidro (Cicarelli p.a.) en agua deionizada al 7,5 y 10\% p/v.

Luego, a $30 \mathrm{~g}$ de la solución de alginato se agregó aceite de pomelo, logrando una carga del 2 y $10 \%$. Se formó una emulsión agitando con un homogenizador DLAB, Modelo D-500 a 2000 rpm durante 3 minutos para lograr la distribución homogénea de la emulsión. Se formaron las cápsulas a través de una extrusión con una bomba jeringa APEMA PC 11 UBT, goteando la emulsión sobre la solución de cloruro de calcio a $5 \mathrm{~cm}$ de altura, con caudal de $90 \mathrm{ml} / \mathrm{h}$ y diámetro de salida de la gota de $0,21 \mathrm{~mm}$. Estas variables de proceso se definieron en trabajos anteriores. El tiempo de reticulación fue de 15-30 y 45 minutos. Luego se filtraron las cápsulas y se lavaron con alcohol terbutílico para eliminar el aceite residual y se registró su peso. Se analizó la morfología de las microcápsulas observándolas con microscopio óptico.

\subsection{Cálculo de la eficiencia de microencapsulación}

La eficiencia porcentual de limoneno encapsulado se determinó por cromatografía gaseosa. Se tomó una muestra de $30 \mu \mathrm{l}$ de la solución de lavado de las microcápsulas y se agregó el estándar interno (metilisobutilcetona) para luego ser analizada con un SHIMADZU GC 14B, con columna polar Megabore DB-WAX P/N 125-7032 de 30m de longitud x $0,53 \mathrm{~mm}$ de diámetro interno $\mathrm{x} 1 \mu$ de espesor, conectada a un detector FID. La temperatura del detector fue de $220^{\circ} \mathrm{C}$ y la temperatura de inyector de $180^{\circ} \mathrm{C}$. La temperatura de la columna se mantuvo isotérmica en $40^{\circ} \mathrm{C}$ durante 2 minutos, luego inició una rampa de $5^{\circ} \mathrm{C}$ Cmin -1 hasta llegar a $180^{\circ} \mathrm{C}$, por último se mantuvo isotérmica a $180^{\circ} \mathrm{C}$ durante 2 minutos. Se comparan las relaciones de áreas de los cromatogramas en una curva de calibración para conocer la masa de aceite encapsulado. El valor de la eficiencia (E\%) se determinó con la siguiente Ec. (1).

$$
\mathrm{E} \%=\mathrm{w} 1 / \mathrm{w} 2100
$$

Donde w1 es la masa de aceite en una masa conocida de microcápsulas que se determina por diferencia de w2 (masa inicial teórica de aceite agregada) y la masa final calculada por cromatografía gaseosa.

Cálculo del rendimiento de microencapsulación. Para determinar el rendimiento, se pesaron las microcápsulas (wm) obtenidas y conociendo la cantidad de principio activo (wa) y de polímero (wp) que se empleó para preparar la emulsión, se aplicó la siguiente Ec. (2):

$$
\mathrm{R} \%=\mathrm{wm} /(\mathrm{wa}+\mathrm{wp}) 100
$$

\subsection{Observación microscópica}

Se analizó la forma y tamaño de las microcápsulas obtenidas con microscopio óptico trinocular marca Lancet modelo SME F6E, equipado con cámara (TOUPCAM UCMOS01300KPA, modelo TP601300A, origen China) y software de procesamiento de datos (Toup View: Touptek Photonics of Toupteck Corporation) con aumento de 4x. 
Diseño experimental. Se utilizó un diseño experimental factorial de dos factores con tres niveles $\left(3^{2}\right)$, con un total de 18 tratamientos para la realización de los experimentos. Se estudiaron como factores la concentración de cloruro de calcio y el tiempo de reticulación, y se analizaron como variables respuesta a la eficiencia y el rendimiento de encapsulación. Este tipo de diseños permite evaluar el efecto de ambos factores en la respuesta del proceso, y determinar si la actuación de ambos es independiente. El modelo matemático de optimización que rige para este modelo de dos factores totalmente aleatorizado es el siguiente, Ec. (3), obtenido a partir de un análisis de regresión:

$$
y=\beta 0+\beta 1 \times 1+\beta 2 \times 2+\beta 12 \times 1 \times 2+\beta 11 \times 12+\beta 22 \times 22+\varepsilon
$$

Donde y representa cada respuesta a analizar, $\beta$ son los efectos medios, $\mathrm{x} 1 \mathrm{y}$ x 2 son los factores o variables de entrada, y $\varepsilon$ es el error medio aleatorio.

Los factores del diseño con sus niveles se pueden observar en la Tabla 1.

Tabla 1. Factores y niveles del diseño experimental

\begin{tabular}{lll}
\hline \multirow{2}{*}{ Niveles } & Factores & \\
\cline { 2 - 3 } & $\mathrm{Cc} \mathrm{CaCl}_{2}(\% \mathrm{p} / \mathrm{v})$ & Tiempo (min) \\
\hline 1 & $\mathrm{a}$ & $\mathrm{b}$ \\
2 & 5 & 15 \\
3 & 10 & 30 \\
\hline
\end{tabular}

\section{Resultados}

Las microcápsulas obtenidas fueron de forma esférica y homogénea y de diámetro de hasta $2 \mathrm{~mm}$. En la Tabla 2 se muestran los valores medios obtenidos de eficiencia y rendimiento de encapsulación para cada factor y nivel, aplicando respectivamente las ecuaciones (1) y (2). Los valores máximos de eficiencia (96,33\%) y rendimiento $(52,01 \%)$ fueron con concentración de cloruro de calcio al 10\% y tiempo de reticulación de 30 minutos. En la Tabla 3 se muestran los resultados de la prueba ANOVA para la eficiencia y rendimiento de encapsulación. Se observa que, tanto la concentración de cloruro de calcio y el tiempo de reticulación, tienen efecto significativo (para $\alpha=0,05)$ sobre ambas variables respuestas. El efecto lineal es mayor que el cuadrático (valor F mayor). En el rendimiento afecta significativamente la concentración de cloruro de calcio y no el tiempo de reticulación. El coeficiente R2 que indica la variabilidad que de los factores que explica el modelo, tiene un valor de 79,20\% para la eficiencia y un 78,61\% para el rendimiento. Las Ecuaciones (4) y (5) muestran las optimizaciones obtenidas para la eficiencia (E\%) y el rendimiento $(\mathrm{R} \%)$ de encapsulación basadas en un modelo de regresión cuadrático. En ambas puede observarse el efecto positivo de los factores concentración de cloruro de calcio (x1), sin embargo, el tiempo de reticulación (x2) posee un efecto positivo sobre la Eficiencia (E\%) y negativa sobre el Rendimiento (R\%). Además el efecto de las interacciones de ambos factores es negativo en ambos casos.

$$
\begin{aligned}
& \mathrm{E} \%=89,68+0,253 \times 1+0,271 \times 2-0,008 \times 1 \times 2-0,0136 \times 12-0,003 \times 22 \\
& \mathrm{R} \%=26,600+4,250 \times 1-0,060 \times 2-0,003 \times 1 \times 2-165 \times 12+0,000 \times 22
\end{aligned}
$$

Las gráficas de contorno muestran que la eficiencia (Fig. 1.a) alcanza valores mayores para concentraciones de cloruro de calcio entre el 9 y $10 \%$ p/v y tiempos entre 25 y 45 minutos. Mientras que el rendimiento (Fig. 1.b) muestra valores mayores para todos los tiempos también con concentraciones de cloruro de calcio entre 9 y $10 \%$ p/v. La optimización de ambas variables a través de la función deseabilidad se muestra en la Fig. 2. La deseabilidad compuesta $(0,83)$ es buena, lo que indica que la combinación de los valores de las variables permite maximizar la eficiencia y el rendimiento. Sin embargo, la deseabilidad individual indica que la configuración es más efectiva para maximizar la eficiencia $(d=0,94)$ que para maximizar el rendimiento $(d=0,74)$. Según el modelo, con una concentración de cloruro de calcio de $10 \%$ p/v y un tiempo de reticulación de 45 minutos se obtendría un rendimiento de 51,7\% y un 96,5\% de eficiencia del proceso de encapsulación. Con estos valores configurados se obtiene un intervalo de predicción con un nivel de confianza del $95 \%$ para la eficiencia de $95,8 \%$ a $97,1 \%$ y para el rendimiento de $46,1 \%$ a 57,4\%. La validación del modelo fue realizada con cinco corridas en el punto óptimo, reticulando con una solución de cloruro de calcio de 
$10 \% \mathrm{w} / \mathrm{v}$, durante un tiempo de 45 minutos. Los resultados obtenidos se ajustaron al modelo, siendo la eficiencia media de $95,89 \pm 0,04 \%$, y el rendimiento medio de $56,87 \pm 1,2 \%$

Tabla 2. Medias de Eficiencia y Rendimiento de encapsulación.

\begin{tabular}{llllr}
\hline & \multicolumn{2}{l}{ Eficiencia (\%) } & \multicolumn{2}{l}{ Rendimiento (\%) } \\
\hline Fuente & Media & DST & Media & DST \\
Cl2Ca & & & & \\
2 & 94,78 & 0,22 & 32,87 & 2,32 \\
5 & 95,04 & 0,22 & 42,43 & 2,32 \\
10 & 96,01 & 0,22 & 51,76 & 2,32 \\
Tiempo & & & & \\
15 & 94,36 & 0,22 & 43,00 & 2,32 \\
30 & 95,71 & 0,22 & 42,35 & 2,32 \\
45 & 95,76 & 0,22 & 41,70 & 2,32 \\
Cl2Ca *Tiempo & & & & \\
$2 * 15$ & 92,98 & 0,37 & 33,39 & 4,02 \\
$2 * 30$ & 95,51 & 0,37 & 32,91 & 4,02 \\
$2 * 45$ & 95,85 & 0,37 & 32,29 & 4,02 \\
$5 * 15$ & 94,53 & 0,37 & 43,68 & 4,02 \\
$5 * 30$ & 95,30 & 0,37 & 42,13 & 4,02 \\
$5 * 45$ & 95,28 & 0,37 & 41,46 & 4,02 \\
$10 * 15$ & 95,57 & 0,37 & 51,93 & 4,02 \\
$10 * 30$ & 96,33 & 0,37 & 52,01 & 4,02 \\
$10 * 45$ & 96,14 & 0,37 & 51,34 & 4,02 \\
\hline
\end{tabular}

Tabla 3. Tabla ANOVA

\begin{tabular}{llllll}
\hline & \multicolumn{3}{l}{ Eficiencia } & \multicolumn{2}{l}{ Rendimiento } \\
\hline Fuente & $\mathrm{GL}$ & $\mathrm{F}$ & $\mathrm{P}$ & $\mathrm{F}$ & $\mathrm{P}$ \\
\hline Modelo & 5 & 9,14 & 0,00 & 8,82 & 0,00 \\
Lineal & 2 & 14,83 & 0,00 & 22,03 & 0,00 \\
$\mathbf{C l}_{\mathbf{2}} \mathbf{C a}$ & 1 & 14,02 & 0,00 & 43,87 & 0,00 \\
Tiempo & 1 & 15,63 & 0,00 & 0,2 & 0,67 \\
Cuadrado & 2 & 2,88 & 0,10 & 0,49 & 0,62 \\
$\mathbf{C l}_{\mathbf{2}} \mathbf{C a} * \mathbf{C l}_{\mathbf{2}} \mathbf{C a}$ & 1 & 0,5 & 0,49 & 0,98 & 0,34 \\
$\quad$ Tiempo*Tiempo & 1 & 5,26 & 0,04 & 0,00 & 1,00 \\
Interacción de 2 factores & 1 & 6,86 & 0,02 & 0,01 & 0,92 \\
$\mathbf{C l}_{2} \mathbf{C a} *$ Tiempo & 1 & 6,86 & 0,02 & 0,01 & 0,92 \\
Error & 12 & & & & \\
$\mathrm{R}^{2}$ & & $79,20 \%$ & & $78,61 \%$ & \\
Falta de ajuste & 3 & 1,71 & 0,23 & 0,02 & 1,00 \\
Error puro & 9 & & & & \\
Total & 17 & & & & \\
\hline
\end{tabular}
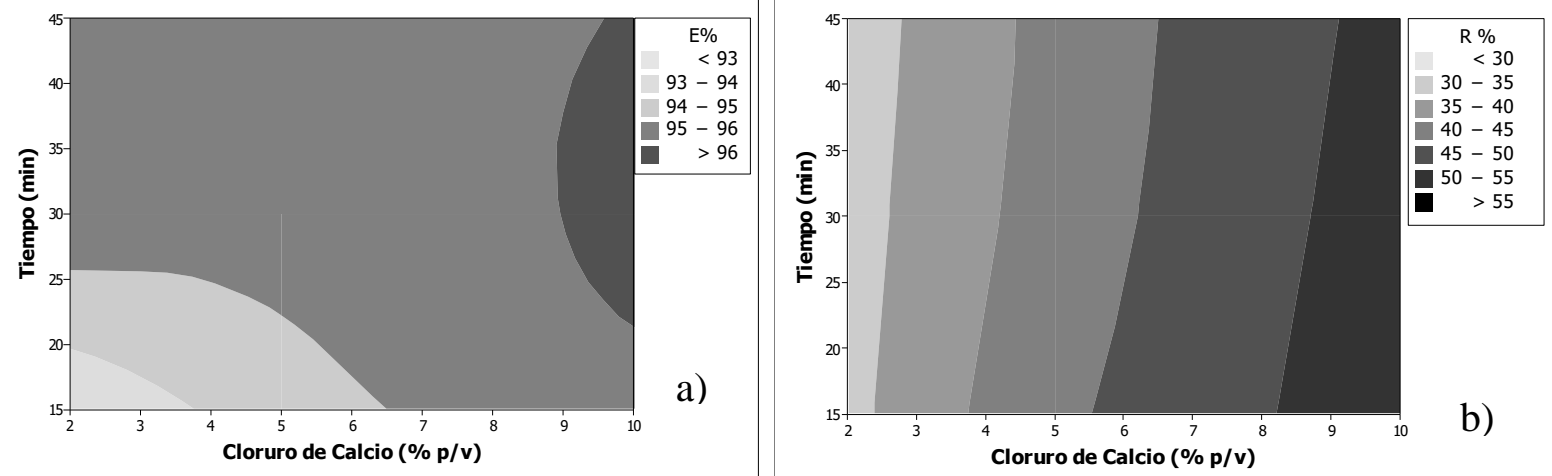

Fig. 1. Gráficas de Contorno: a) eficiencia de encapsulación, b) rendimiento de encapsulación 


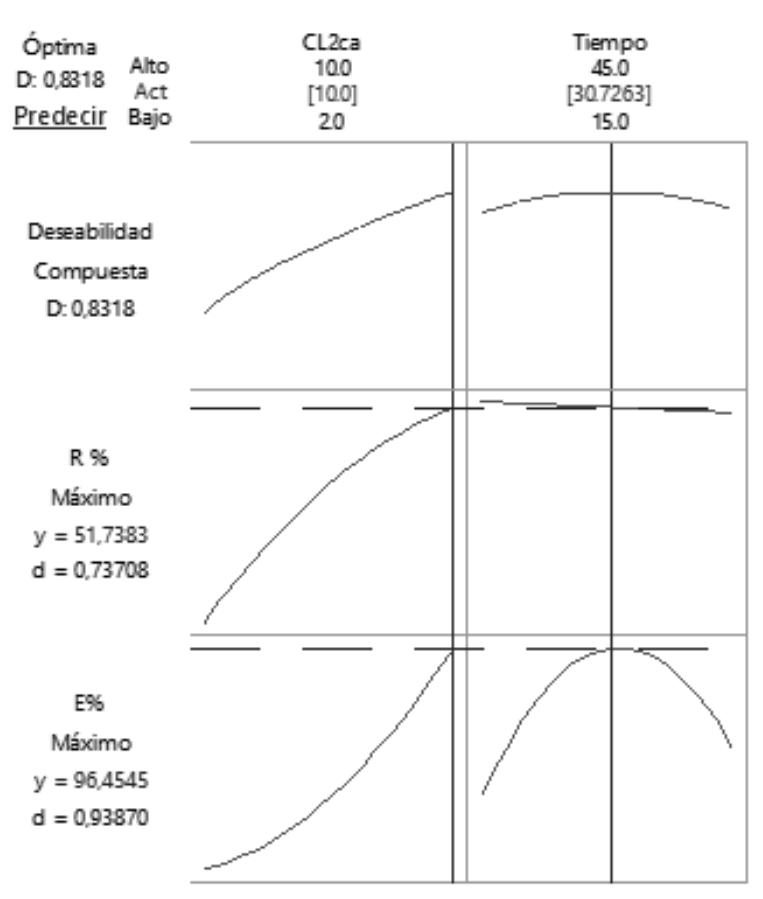

Fig. 2. Gráfica de optimización y deseabilidad

\section{Discusión:}

En estudios similares se reportaron eficiencias menores (85\%) que las obtenidas, con concentración de cloruro de calcio de 1,5\% p/v (Benavides et al., 2016); y eficiencias del 83,8\% con el uso de un agente emulsionante (Tween-80) (Banerjee et al., 2013). El rendimiento máximo obtenido es similar al reportado por Benavides et al. (2016) de un 60 a 62\%. En otro estudio de Ribeiro et al. (1999) se obtuvieron rendimientos del 60-80\% con el uso de emulsiones dobles de aceite y agua, además de la incorporación de quitosano, aumentando la complejidad del proceso de esferificación.

\section{Conclusión}

Se estudió la encapsulación de aceite de pomelo por gelificación iónica externa y extrusión con alginato al 1\% p/p y carga de aceite del $2 \% \mathrm{p} / \mathrm{p}$. Se optimizó el proceso de obtención de las microcápsulas obteniendo el punto óptimo cuando la concentración de cloruro de calcio es del 10\% p/v con tiempo de reticulación de 45 minutos. En estas condiciones la eficiencia media fue de $95,89 \pm 0,04 \%$, y el rendimiento medio de $56,87 \pm 1,2 \%$.

\section{Referencias}

Banerjee, S., Chattopadhyay, P., Ghosh, A., Goyary, D., Karmakar, S. y Veer, V. (2013). Influence of process variables on essential oil microcapsule properties by carbohydrate polymer-protein blends. Carbohydrate polymers, 93(2), 691697. doi.org/10.1016/j.carbpol.2013.01.028

Benavides, S., Cortés, P., Parada, J. y Franco, W. (2016). Development of alginate microspheres containing thyme essential oil using ionic gelation. Food chemistry, 204, 77-83. doi.org/10.1016/j.foodchem.2016.02.104

Jun-xia, X., Hai-yan, Y. y Jian.Y. (2011). Microencapsulation of sweet orange oil by complex coacervation with soybean protein isolate/gum Arabic. Food Chemistry, 125, 1267-1272. doi.org/10.1016/j.foodchem.2010.10.063

Goh, C. H., Heng, P. W. S. y Chan, L. W. (2012). Alginates as a useful natural polymer for microencapsulation and therapeutic applications. Carbohydrate Polymers, 88(1), 1-12. doi.org/10.1016/j.carbpol.2011.11.012

Hosseini, S., Hosseini, H., Mohammadifar, M., Mortazavian, A., Mohammadi, A., Khosravi-Darani, K., Shojaee-Aliabadi, S. y Khaksar, R. (2013). Incorporation of essential oil in alginate microparticles by multiple emulsion/ionic gelation process. International Journal of Biological Macromolecules, 62, 582-588. doi.org/10.1016/j.ijbiomac.2013.09.054 
Lupo Pasin, B., González Azón, C. y Maestro Garriga, A. (2012). Microencapsulación en alginato para alimentos. Tecnologías y aplicaciones. Revista Venezolana de Ciencia y Tecnología de Alimentos, 3(1), 130-151.

Pasukamonset, P., Kwon, O. y Adisakwattana, S. (2016). Alginate-based encapsulation of polyphenols from Clitoria ternatea petal flower extract enhances stability and biological activity under simulated gastrointestinal conditions. Food Hydrocolloids, 61, 772-779. doi.org/10.1016/j.foodhyd.2016.06.039

Ribeiro, A. J., Neufeld, R. J., Arnaud, P. y Chaumeil, J. C. (1999). Microencapsulation of lipophilic drugs in chitosancoated alginate microspheres. International journal of pharmaceutics, 187(1), 115-123. doi.org/10.1016/S03785173(99)00173-8

Smidsrod, O. y Draget, KL (1996). Chemistry and physical properties of alginates. Carbohydrate Eur 14:6-13.

Vasek, O. M., Cáceres, L. M., Chamorro, E. R., \& Velasco, G. A. (2015). Antibacterial activity of Citrus paradisi essential oil. Journal of Natural Products, 8, 16-26. 